\title{
BMJ open Assessing the knowledge of sudden unexpected death in the young among Canadian medical students and recent graduates: a cross-sectional study
}

\author{
Felicity F Huisma, James E Potts, Karen A Gibbs, Shubhayan Sanatani
}

To cite: Huisma FF, Potts JE, Gibbs KA, et al. Assessing the knowledge of sudden unexpected death in the young among Canadian medical students and recent graduates: a cross-sectional study. BMJ Open 2012;2: e001798. doi:10.1136/ bmjopen-2012-001798

- Prepublication history and additional material for this paper are available online. To view these files please visit the journal online (http://dx.doi.org/10.1136/ bmjopen-2012-001798).

Received 4 August 2012 Revised 4 August 2012 Accepted 22 November 2012

This final article is available for use under the terms of the Creative Commons Attribution Non-Commercial 2.0 Licence; see http://bmjopen.bmj.com

Division of Pediatric Cardiology, Department of Pediatrics, British Columbia Children's Hospital, University of British Columbia, Vancouver, Canada

Correspondence to Dr Felicity Huisma; fhuisma@ cw.bc.ca

\section{ABSTRACT}

Objective: To determine the level of knowledge concerning Sudden Unexpected Death in the Young (SUDY) among Canadian medical students and recent graduates ( $\leq 5$ years after graduating).

Design: A cross-sectional study was conducted by distributing a standardised, multiple choice, online questionnaire which assessed basic knowledge of SUDY. Setting: Canadian medical schools and residency training programmes.

Participants: 614 Canadian medical students (in either their penultimate or final year) and recent graduates ( $\leq 5$ years after graduating) completed an anonymous online questionnaire.

Primary and secondary outcome measures: The level of knowledge regarding molecular aetiology, clinical presentation, pharmacological management and modes of inheritance of six of the commonest conditions causing SUDY, including hypertrophic cardiomyopathy (HCM), arrhythmogenic right ventricular cardiomyopathy (ARVC), Brugada syndrome, catecholaminergic polymorphic ventricular tachycardia (CPVT), long QT syndrome (LQT) and Wolff-Parkinson White syndrome (WPW), were compared between medical students and recent graduates. Questions were broken down into basic knowledge and advanced categories and analysed as a secondary outcome measure.

Results: 0 f 614 responses, approximately two-thirds were answered by recent graduates, who generally scored $10 \%$ higher on all subject categories than medical students. Overall, questions regarding HCM were best answered (40\%), followed by WPW syndrome (32\%), CPVT $(30 \%)$, ARVC $(23 \%)$, Brugada syndrome $(21 \%)$ and LQT syndrome (17\%). Questions categorised as basic knowledge were answered $30 \%$ and $39 \%$ correctly in medical student and recent graduate groups, respectively, and those in the advanced category were answered $20 \%$ and $25 \%$ correctly.

Conclusions: Survey respondents fared poorly when answering questions regarding SUDY, which may be a reflection of inadequate medical education regarding these disorders. Standardised teaching regarding SUDY needs to occupy a stronger focus in Canadian medical curricula in order to prevent more unnecessary deaths by these syndromes in the future.

\section{ARTICLE SUMMARY}

Article focus

- Arrhythmic aetiologies play a significant role in sudden death in individuals under 35 , and a majority of these disorders are inherited in an autosomal-dominant fashion.

- Despite the high degree of heritability that these disorders exhibit, first and second-degree relatives of Sudden Unexpected Death in the Young (SUDY) victims are generally under-referred for cardiological screening, putting them at risk of death from the same disorder.

- We set out to determine the current level of knowledge regarding SUDY disorders among Canadian final-year medical students and recent graduates ( $\leq 5$ years after graduating).

\section{Key message}

- Canadian medical students and recent graduates fared poorly when answering questions regarding SUDY, which illustrates a need for more education and awareness regarding these disorders.

Strengths and limitations of this study

- The number of responses collected for this survey was high, and respondents were well distributed according to geographical location as well as years of study.

- Voluntary surveys always employ a degree of response bias, where those who answer may be more knowledgeable, or more interested in the topics discussed, than the general population.

\section{INTRODUCTION}

The sudden death of a young person is a rare and tragic event, which has a significant impact on a family and the community. Arrhythmic aetiologies play a considerable role in sudden cardiac death in all age groups, with it occupying a greater proportion of paediatric deaths due to the rise of coronary artery disease in older age cohorts. ${ }^{1}{ }^{2}$ These arrhythmic aetiologies can be broken down into structural or primary 
electrical heart disease, and form a phenomenon known as Sudden Unexpected Death in the Young (SUDY). ${ }^{3}$

Structural heart disease in the context of SUDY refers to cardiomyopathies, which include hypertrophic cardiomyopathy (HCM) and arrhythmogenic right ventricular cardiomyopathy (ARVC). ${ }^{4}$ Primary electrical disease refers to ion channelopathies such as long QT syndrome, Brugada syndrome and catecholaminergic polymorphic ventricular tachycardia (CPVT), as well as other rare conditions such as short QT syndrome. ${ }^{45}$ Aetiologies of these diseases can be linked to genetic mutations in proteins that control either the contractile or electrical activity of the heart and many of these mutations display monogenic inheritance patterns, with first-degree relatives inheriting the same disorders as frequently as $25-50 \% .^{15-11}$

Conditions associated with SUDY are becoming increasingly recognised as knowledge of these disorders advances. Ion channelopathies are now known to account for almost $20 \%$ of sudden infant death syndrome fatalities. ${ }^{12-15}$ Many of these conditions cannot be readily diagnosed upon autopsy or even clinical evaluation. ${ }^{3}{ }^{16}$ Therefore, when someone dies suddenly from unexplained causes, usually SUDY or idiopathic ventricular fibrillation are suspected. ${ }^{17}$

Due to the high degree of heritability of these disorders, it is imperative that family members of SUDY victims be properly assessed. ${ }^{18-22}$ Systematic review of individuals experiencing cardiac arrest and their firstdegree relatives has been shown to identify a cause in as many as $44 \% .{ }^{62} 23-28$ Often, a general practitioner functions as the first point of contact for individuals with a family history of sudden unexplained death, and fulfils the important role of generating referrals to specialists. ${ }^{29}$ In addition, the highly publicised nature of SUDY makes general practitioners important players in the education of families and communities. ${ }^{30}$ Despite this, a general lack of referrals has been well documented. ${ }^{31}$ A possible cause for this disparity is a knowledge gap among medical professionals concerning inherited arrhythmia substrates, presumably stemming from a deficit in the education that medical students receive throughout their training.

A lack of consensus about minimum acceptable standards in cardiology principles and skills for students and doctors has been an ongoing debate, particularly with regard to ECG interpretation and lead placement. ${ }^{32} 33$ This is well demonstrated in a study by Hill $e t a l,{ }^{34}$ which recited a detection rate of only $70 \%$ when paediatric electrophysiologists were presented with pathological preparticipation screening ECGs. Similarly, other studies evaluating correct lead placement among healthcare professionals have shown a disappointing degree of disparity, with cardiologists often faring the poorest. ${ }^{35} 36$ Therefore, it seems that education in cardiology concepts is variable, and inadequate in some cases. To the best of our knowledge, no studies have been conducted to determine the level of proficiency of graduating medical students in answering questions regarding SUDY conditions.

In order to gain a better understanding of the current level of knowledge of Canadian medical students and recent graduates, a standardised, online survey was distributed in which students from all 14 Canadian medical schools participated. The survey tested basic knowledge and understanding of six arrhythmia substrates that are commonly involved in SUDY, including ARVC, Brugada syndrome, CPVT, HCM, long QT syndrome and Wolff-Parkinson-White (WPW) syndrome.

\section{METHODS}

A questionnaire was designed to test basic knowledge concepts about cardiac disorders that are commonly associated with SUDY. The survey was designed using surveymonkey.com (SurveyMonkey, LLC, Palo Alto, California, USA) and was distributed to any Canadian medical student in their penultimate or final year, or within 5 years after graduating. Respondents who did not meet any of these criteria were immediately excluded. To distribute the surveys, undergraduate and postgraduate medical offices from 14 Canadian medical schools were contacted and asked to distribute the link to the online questionnaire to their students. Reminder emails were then sent again 5 months later to recruit non-respondents. Students were informed at the beginning of the survey that their participation was voluntary and anonymous.

Demographic information was collected from each respondent, which included their intended or current specialty, which university they were enrolled in or had graduated from, and, where applicable, under which university they were completing their residency training.

The survey consisted of a total of 31 questions. Questions were designed with the help of an electrophysiologist, and standardisation was sought by distributing it to three experts in the field of electrophysiology, two of whom scored $100 \%$ and one who answered one question incorrectly (which was regarding the increased incidence of ARVC in Newfoundland, Canada, and can be explained by the fact that this respondent was educated and currently practices in the USA). Questions can be broken down into eight categories: general concepts, ARVC, Brugada syndrome, CPVT, HCM, long QT syndrome, WPW and genetic principles. For each category, between three and five multiple choice questions were asked, which aimed to assess general knowledge about the underlying molecular aetiology of each disorder, the clinical presentation, pharmacological management and basic knowledge (which tested well-known facts about the disorder where applicable, such as where the disorder was most prevalent, or dynamic manoeuvres that would assist in diagnosis). General questions aimed to assess basic knowledge about the causes of SUDY, and included questions such as whether the disorders mentioned would be identifiable upon autopsy. Finally, 
genetic principles focused on the commonest modes of inheritance of each of the six disorders tested.

The use of multiple choice and true/false questions was based largely on logistical factors, recognising that to analyse over 600 short-answer or long-answer questions objectively would have been challenging. In addition, the vast majority of written examinations in Canadian medical schools are in multiple-choice format. For example, the Medical Council of Canada Qualifying Examinations, which are required from all graduating Canadian medical students and residents in order to obtain licensure, is written entirely in multiple-choice format. ${ }^{37}$

All the questions were also divided into two categories: basic knowledge, which were felt to be clinically relevant questions which tested the basic knowledge of SUDY disorders that present relatively commonly, and advanced questions, which we did not expect the majority to answer correctly, but were designed to assess the level of knowledge among medical students and recent graduates. (The basic knowledge questions are marked with an asterisk in online supplementary appendix 1.)

Where data were missing, it was still included in the calculation of results. For example, incomplete responses were still used in the denominator to calculate average scores. In addition, since it was impossible to discern at which point in the survey some respondents had dropped out, they were also kept in the analysis. This was justified in light of the fact that medical students are generally well aware that incomplete answers will be marked as incorrect.

Approval from the University of British Columbia's Behavioural Research Ethics Board and Children's and Women's Health Centre of British Columbia's Research Review Committee was obtained prior to beginning the study.

\section{DATA ANALYSIS}

The proportion of correct answers for each question was compared between the medical student and recent graduate groups, and these results are presented in the survey questions document (see online supplementary appendix 1). For further analysis, all the questions were broken down into two sets of categories, one which was based on the cardiac disease, the second based on the type of question asked (such as general knowledge, aetiology, clinical presentation, treatment or inheritance mode).The proportion of correct answers for each question were then compared and plotted as frequency charts for each category (see figures 1 and 2).

\section{RESULTS}

Between September 2011 and March 2012, 748 respondents began the survey. In total 134 were immediately excluded because they were not in their penultimate or final year of medical school, were not within 5 years of graduating, or they did not attend a Canadian medical

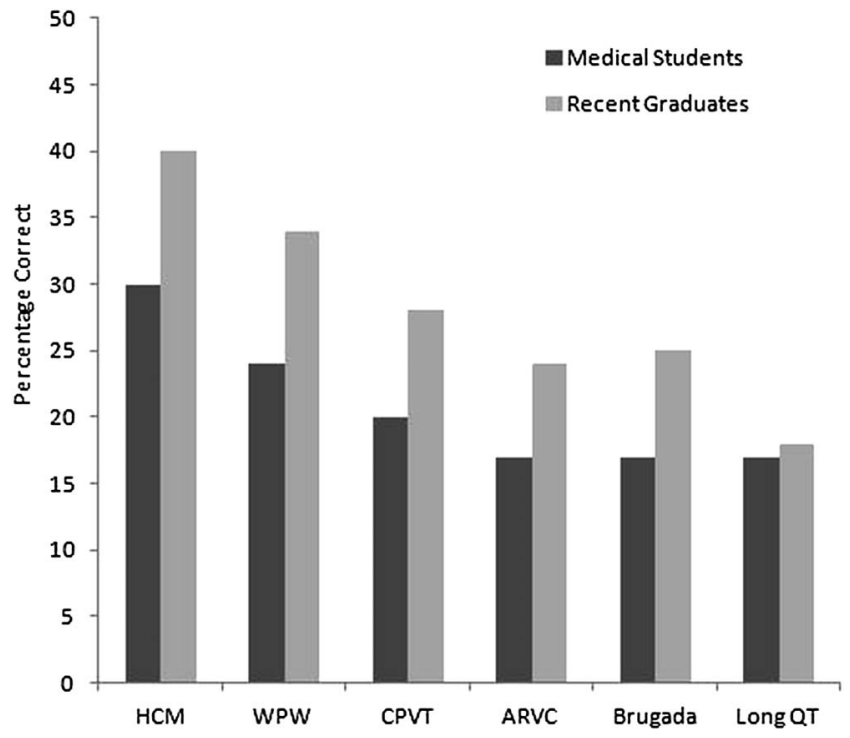

Figure 1 Proportion of questions answered correctly by medical students and recent graduates $(\leq 5$ years after graduating) according to syndrome. ARVC, arrhythmogenic right ventricular cardiomyopathy; Brugada, Brugada syndrome; CPVT, catecholaminergic polymorphic ventricular tachycardia; HCM, hypertrophic cardiomyopathy; Long QT, long QT syndrome; WPW, Wolff-Parkinson-White syndrome.

school. Of the remaining 614 respondents, 468 finished the survey (ie pressed the 'finish' button at the end of the last page), and an average of 335 respondents answered each question, resulting in a completion rate of $54 \%(335 / 614)$.

\section{Demographics of respondents}

Despite the fact that not all Canadian medical schools agreed to distribute the survey to their students, all 14

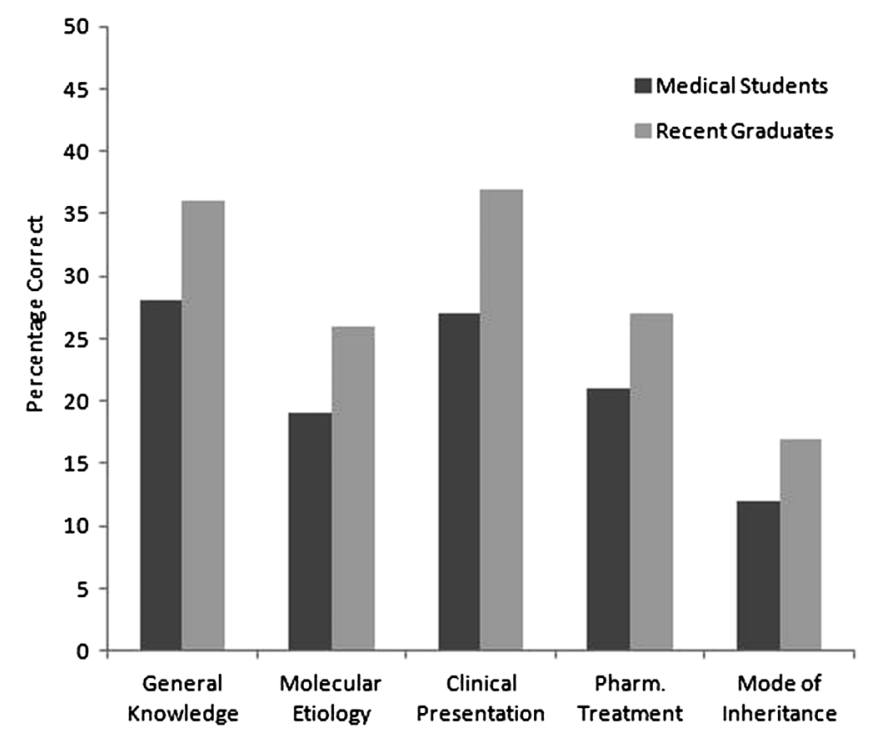

Figure 2 Proportion of questions answered correctly by medical students and recent graduates according to topic category. Pharm, pharmacological. 
Canadian medical schools were represented in the study, as Canadian postgraduate students often complete their residency training through a university other than from which they graduated.

Of the 614 respondents, 398 were recent graduates (making up 65\% of the sample) and 216 were medical students (35\% of the sample). These numbers were employed as the denominators for all calculations.

When asked which specialty they would like to pursue or were in the process of pursuing, respondents most commonly answered family medicine/general practice (31/27\% for medical students/recent graduates, respectively), paediatrics $(9 / 10 \%)$ and internal medicine (14/ $8 \%)$. Cardiology ranked tenth with $1 / 2 \%$ of students choosing this as their specialty of choice.

\section{Knowledge of arrhythmia substrates}

On average, recent graduates (having graduated $\leq 5$ years ago) fared better on all survey topics than medical students, by generally answering between $5 \%$ and $15 \%$ more accurately on all subject categories (see figures 1 and 2).

When knowledge regarding the six specific disorders was tested, questions regarding HCM were best answered (with an average of $35 \%$ of questions answered correctly by medical student respondents and $45 \%$ by recent graduates), followed by WPW, CPVT, ARVC, Brugada syndrome and finally, long QT syndrome, the latter topic being answered correctly $18 \%$ of the time by both student populations (see figure 1). Questions regarding molecular aetiology and pharmacological treatment were answered less accurately than general questions or those concerning clinical presentation/diagnosis (with an average difference of $10 \%$, see figure 2).

\section{Basic knowledge questions}

Of the medical student and recent graduate groups, $30 \%$ and $39 \%$ of the questions categorised as basic knowledge were answered correctly, respectively. Forty-two per cent and $48 \%$ correctly answered that HCM is the most common cause of sudden cardiac death in athletes $\leq 35$ years old. Between $10 \%$ and $45 \%$ of medical students and $50-60 \%$ of recent graduates correctly answered that HCM and myocardial infarction would have positive findings on autopsy, and that LQT, Brugada syndrome and CPVT would not. Thirty-three per cent and $42 \%$ of respondents correctly identified that the accessory pathway in WPW connects the atrium to the ventricle. Less than $17 \%$ of medical students, and under $26 \%$ of recent graduates, correctly identified the most common modes of inheritance (autosomal dominant) of LQT, Brugada syndrome, CPVT, HCM and ARVC. Forty-two per cent and 53\% correctly identified the inheritance rate of an autosomal-dominant disease. Thirty per cent and $38 \%$ of both groups correctly identified the difference between penetrance and expressivity.

\section{Advanced questions}

On average, $20 \%$ and $25 \%$ of medical students and recent graduates, respectively, answered these questions correctly. Fourteen per cent and 19\% correctly identified the upper limit of a normal corrected QT interval in men and women, and $12 \%$ and $14 \%$ of respondents in both groups correctly identified the calculation used to correct the QT interval. Thirty-eight per cent and $43 \%$ of respondents from both respective groups correctly answered that rapid access to defibrillation was the most effective life-saving measure during a cardiac arrest. Ten per cent and $14 \%$ correctly identified sleep to be the most likely time for a patient with Brugada syndrome to experience sudden cardiac death.

Many questions in this category asked the molecular mechanism of the disorders tested, which were generally answered correctly by under a quarter of respondents. For example, $11 \%$ and $13 \%$ of respondents in both groups correctly identified desmosomes to be abnormal in ARVC, $16 \%$ and $25 \%$ correctly identified an abnormality in calcium conduction to be the underlying aetiology in CPVT. Another theme in this category asked about methods as well as signs used to diagnose these conditions, or the pharmacological management of them. For example, $28 \%$ and $41 \%$ of respondents correctly identified bidirectional ventricular tachycardia during and exercise stress test to be diagnostic of CPVT. Twenty-two per cent and 29\% of respondents correctly identified verapamil to be contraindicated in patients with WPW, and $16 \%$ and $22 \%$ correctly answered sodium channel blockers to be contraindicated in Brugada syndrome. Twenty-four per cent and 30\% correctly answered an ECG to be the most reliable diagnostic test for Brugada syndrome.

\section{DISCUSSION}

The explosion of knowledge in the field of medicine in the last two decades has overwhelmed the scope of what can be covered in medical training. However, some education on SUDY is likely to save lives. It is clear that Canadian medical students do not receive enough education regarding these conditions, with medical students answering an average of $25 \%$ of questions correctly, followed by $34 \%$ of recent graduates (having graduated $\leq 5$ years ago). Although it is encouraging that recent graduates answered more accurately on all topic categories, the overall level of knowledge is still quite low.

Many of the questions in this survey are difficult. The question set was divided into basic knowledge and advanced categories, the former of which we felt the majority (at least 50\%) should be able to answer and concerned basic electrophysiology concepts as well as conditions which present more frequently relative to some of the other SUDY disorders. The questions categorised as advanced were designed to better determine the knowledge level among current graduates. 
What we were surprised to discover was that still only $30 \%$ and $39 \%$ of questions in the basic knowledge category were answered correctly by the medical student and recent graduate groups, respectively. These results are concerning and cause us to speculate that in some cases, current graduating medical students have not even heard of some of these conditions. Although proper pharmacological management of Brugada syndrome may not be imperative for a general practitioner to properly refer a relative of a SUDY victim for screening, the recognition of the existence of these disorders, combined with an appreciation for their high rates of heritability, certainly are. What this survey clearly demonstrates is a concerning lack of knowledge regarding these disorders altogether.

Electrophysiology has historically been a topic for residents and fellows seeking specialised training in cardiology. The Canadian medical curriculum is not standardised nationally, although qualifications for licensure later are. ${ }^{38}$ Therefore, although it is difficult to pinpoint how much education Canadian medical students receive or should be receiving on the topic, what this survey demonstrates is that it is arguably not enough.

A concerning result of this study is the lack of knowledge regarding the commonest modes of inheritance of SUDY disorders. Long QT syndrome, Brugada syndrome, CPVT, HCM and ARVC most commonly demonstrate autosomal-dominant inheritance patterns. ${ }^{3} 11 \quad 14$ Additionally, the bulk of these disorders, particularly the channelopathies, are not diagnosable upon autopsy. ${ }^{39}$ Therefore, a patient who presents with a positive family history of a first or second-degree relative who died suddenly of an unknown cause should immediately alert the physician to the possibility of a silent arrhythmia. Furthermore, many patients with these disorders display sentinel symptoms, particularly syncope, awarding the physician yet another chance to diagnose the disorder early. ${ }^{1624}$

A majority (approximately 30\%) of respondents in this survey stated family medicine/general practice to be their intended or designated specialty. Family physicians generally function as the first respondent for any medical issue, and they also generate the bulk of referrals to specialists. ${ }^{29}$ Therefore, a good knowledge base, combined with a high degree of suspicion for arrhythmia disorders, is imperative in order to make these diagnoses in time.

Diagnosis of these disorders is generally difficult. ${ }^{40}$ Many of them are silent on an ECG, and require echocardiography, exercise-stress testing, pharmacological testing, or in some cases, genetic testing, to properly diagnose them. ${ }^{41-44}$ This speaks to the need for referral to specialised heritable arrhythmia clinics that are expert in diagnosing and treating these disorders. Treatment is generally straight-forward, and requires pharmacological therapy, or in more severe cases, transcatheter ablation or insertion of an implantable cardioverter-defibrillator. ${ }^{45-47}$ Those who are managed correctly go on to lead productive lives, and are generally unhindered in their activities of daily living. ${ }^{48}$

\section{Study limitations}

A study such as this one, which involves administering a voluntary survey, inherently suffers from limitations. Although it was impossible to discern a response rate (because we were unable to determine exactly how many surveys were distributed), we can assume it to be relatively low (about $20 \%$ if it follows trends similar to other surveys distributed to the medical community.) Because the survey was voluntary, we were unable to practice random sampling. We can therefore presume that the results suffer from a level of voluntary response bias, where those who participated were likely more knowledgeable or at least more interested in the topics discussed, than non-respondents.

In addition, the survey was not administered to a test group in order to develop reliability and validity of the questions before distributing it to the sample population.

\section{CONCLUSION}

The current knowledge of SUDY among Canadian medical students and recent graduates is poor, which may be a reflection of current medical education in this field. Increased awareness and education regarding SUDY are important in order to prevent further deaths by these treatable disorders in the future.

Acknowledgements The authors would like to thank all of the medical students and residents who took the time to complete our survey, as well as the undergraduate and postgraduate offices that helped us by distributing the survey link to their students on our behalf.

Contributors FH was responsible for designing the research project and the survey questions, data-collection, as well as for drafting and revising the final manuscript. She is the guarantor. SS supervised the project, aided with the initial design of the research as well as the survey questions and revised the final manuscript. JEP and KAG helped with statistical analysis and final review of the manuscript.

Funding This research received no specific grant from any funding agency in the public, commercial or not-for-profit sectors.

Competing interests None.

Data sharing statement We have also attached the original survey in this submission (see online supplementary appendix 1).

\section{REFERENCES}

1. Zeng ZJ, Croft JB, Giles WH, et al. Clinical investigation and reports: sudden cardiac death in the United States, 1989 to 1998. Circulation 2001;104:2158-63.

2. Behr E, Wood DA, Wright M, et al. Cardiological assessment of first-degree relatives in sudden arrhythmic death syndrome. Lancet 2003;362:1457-9

3. Vaartjes I, Hendrix A, Hertogh EM, et al. Sudden death in persons younger than 40 years of age: incidence and causes. Eur $J$ Cardiovasc Rehabil 2009;16:592-6.

4. Van der Werf C, van Langen I, Wilde A. Advances in arrhythmia and electrophysiology, sudden death in the young: what do we know about it and how to prevent? Arrhyth Electrophysiol 2010;3:96-104.

5. Tan HL, Hofman N, van Langen IM, et al. Sudden unexplained death: heritability and diagnostic yield of cardiological and genetic examination in surviving relatives. Circulation 2005;112:207-13. 
6. Dong M, Niklewski PJ, Wang HS. Ionic mechanisms of cellular electrical and mechanical abnormalities in Brugada syndrome. Am J Physiol Heart Circ Physiol 2011;300:H279-87.

7. Roden DM, Balser JR, Geroge AL, et al. Cardiac ion channels. Annu Rev Physio 2002;64:431-75.

8. Tardiff J. Sarcomeric proteins and familial hypertrophic cardiomyopathy: linking mutations in structural proteins to complex cardiovascular phenotypes. Heart Fail Rev 2005;10:237-48.

9. Watkins H, McKenna WJ, Thierfelder L, et al. Mutations in the genes for cardiac troponin $\mathrm{T}$ and alpha-tropomyosin in hypertrophic cardiomyopathy. N Engl J Med 1995;332:1058-64.

10. Hollman A, Goodwin JF, Teare D, et al. A family with obstructive cardiomyopathy (asymmetrical hypertrophy). Br Heart $J$ 1960;22:449-56.

11. Behr ER, Dalageorgou C, Christiansen M, et al. Sudden arrhythmic death syndrome: familial evaluation identifies inheritable heart disease in the majority of families. Eur Heart $J$ 2007;29:1670-80.

12. Ackerman J, Siu B, Sturner W, et al. Postmortem molecular analysis of SCN5A defects in sudden infant death syndrome. JAMA 2001;286:2264-9.

13. Morris JA, Harrison L, Brodison A, et al. Sudden infant death syndrome and cardiac arrhythmias. Future Cardiol 2009;5:201-7.

14. Tester DJ, Ackerman MJ. Sudden infant death syndrome: how significant are the cardiac channelopathies? Cardiovasc Res 2005;67:388-96.

15. Klaver EC, Versluijs GM, Wilders R. Cardiac ion channel mutations in sudden infant death syndrome. Int J Cardiol 2011;152:162-70.

16. Drory $Y$, Turetz $Y$, Hiss $Y$, et al. Sudden unexpected death in persons \&lt;40 years of age. Am J Cardiol 1991;68:1388-92.

17. Hiroshi K, Ohnishi S, Ohtuka M, et al. Idiopathic ventricular fibrillation induced with vagal activity in patients without obvious heart disease. Circulation 1997;95:2277-85.

18. McKoy G, Protonotarios N, Crosby A, et al. Identification of a deletion in plakoglobin in arrhythmogenic right ventricular cardiomyopathy with plasmoplantar keratoderma and woolly hair (Naxos disease). Lancet 2000;355:2119-24.

19. Moss AJ. Long QT syndrome. JAMA 2003;289:2041-4.

20. Maron BJ, Nichols PF III, Pickle LW, et al. Patterns of inheritance in hypertrophic cardiomyopathy: assessment by M-mode and two-dimensional echocardiography. Am J Cardiol 1984;53:1087-94.

21. Weiss R, Barmada MM, Nguyen T, et al. Clinical and molecular heterogeneity in the Brugada syndrome; a novel gene locus on chromosome 3. Circulation 2002;105:707-13.

22. Wilde AAM, Bezzina CR. Genetics of cardiac arrhythmias. Heart 2005;91:1352-8.

23. Behr ER, Casey A, Sheppard M, et al. Sudden arthythmic death syndrome: a national survey of sudden unexplained cardiac death Heart 2007;93:601-5.

24. Krahn A, Healey J, Simpson C, et al. Sentinel symptoms in patients with unexplained cardiac arrest: from the Cardiac Arrest Survivors with Preserved Ejection Fraction registry (CASPER). J Cardiovasc Electrophysiol 2012;23:60-6.

25. Gollob MH, Blier L, Brugada R, et al. Society position statement: recommendations for the use of genetic testing in the clinical evaluation of inherited cardiac arrhythmias associated with sudden cardiac death: Canadian Cardiovascular Society/Canadian Heart Rhythm Society joint position paper. Can J Cardiol 2001;27:232-45.

26. McKenna WJ. Sudden arrhythmic death syndrome: familia evaluation identifies inheritable heart disease in the majority of families. Eur Heart J 2008;29:1670-80.
27. Drezner JA, Fudge J, Harmon KG, et al. Warning symptoms and family history in children and young adults with sudden cardiac arrest. J Am Board Fam 2012;25:408-15.

28. Wisten A, Messner T. Symptoms preceding sudden cardiac death in the young are common but often misinterpreted. Scandinavian Cardiovasc J 2005;39:143-9.

29. O'Donnell K. Variation in GP referral rates: what can we learn from the literature? J Fam Pract 2000;17:462-71.

30. Cooper JJ. Boy, 12, dies months after surviving heart attack. Yahoo Sports. 2012 Apr 23 (accessed 3 May 2012). http://sports.yahoo.com/news/boy-12-dies-months-surviving005849171-spt.html

31. Lim Z, Gibbs K, Potts J, et al. A review of sudden unexpected death in the young in British Columbia. Can J Cardiol 2009;26:22-6.

32. Morrison WG, Swann IJ. Electrocardiograph interpretation by junior doctors. Arch Emerg Med 1990;7:108-10.

33. Little B, Mainie I, Ho KJ, et al. Electrocardiogram and rhythm strip interpretation by final year medical students. Ulster Med J 2001;71:i5

34. Hill AC, Miyake CY, Grady S, et al. Accuracy of interpretation of preparticipation screening electrocardiograms. J Pediatr 2011;159:783-8.

35. Rajageneshan R, ludlam CL, Francis DP, et al. Accuracy in ECG lead placement among technicians, nurses, general physicians, and cardiologists. Int J Clin Practice 2008;62:65-70.

36. McCann K, Holdgate A, Mahammad R, et al. Accuracy of ECG electrode placement by emergency department clinicians. Emerg Med Australasia 2007;19:442-8.

37. Page G, Bordage G. The medical council of Canada's key features project: a more valid written examination $\mathrm{f}$ clinical decision-making skills. J Assoc Am Med Coll 1995;70:104-10.

38. Committe on Accreditation of Canadian Medical Schools (CACMS) http://www.afmc.ca/index-e.php (accessed 4 Oct 2012).

39. Basso C, Burke M, Fornes $\mathrm{P}$, et al. Guidelines for autopsy investigation of sudden cardiac death. Virchows Archiv 2008;452:11-18.

40. Collins K, Dubin A. Detecting and diagnosing arrhythmias in adults with congenital heart disease. Curr Cardiol Rep 1999;5:331-5.

41. Brembilla-Perrot B. Pharmacological testing in the diagnosis of arrhythmias. Minerva Cardioangiol 2010;58:505-17.

42. Cubbon RM, Kahn M, Rajwani A, et al. MT. Echocardiography in the investigation of cardiomyopathy. Ultrasound 2008;15:73-9.

43. Shwartz PJ, Priori SG, Dumaine R, et al. A molecular link between the sudden infant death syndrome and the long-QT syndrome. N Engl J Med 2000;343:262-7.

44. McNair WP, Ku L, Mathew RG, et al. Heart failure: SCN5A mutation associated with dilated cardiomyopathy, conduction disorder, and arrhythmia. Circulation 2004;100:2163-7.

45. Brugada J, Brugada R, Brugada P. Pharmacological and device approach to therapy of inherited cardiac diseases associated with cardiac arrhythmias and sudden death. J Electrocardiol 2000;33:41-7.

46. McRury ID, Haines DE. Ablation for the treatment of arrhythmias. Proc IEEE 1996;84:404-16.

47. Moss AJ, Hall J, Cannom DS, et al. Improved survival with an implanted defibrillator in patients with coronary disease at high risk for ventricular arrhythmia. N Engl J Med 1996;335:1933-40.

48. Marron BJ, Chaitman BR, Ackerman MJ, et al. Recommendations for physical activity and recreational sports participation for young patients with genetic cardiovascular diseases. Circulation 2004;109:2807-16 